This item was submitted to Loughborough's Research Repository by the author.

Items in Figshare are protected by copyright, with all rights reserved, unless otherwise indicated.

\title{
Artificial magnetic conductor surfaces and their application to low-profile high-gain planar antennas
}

PLEASE CITE THE PUBLISHED VERSION

http://dx.doi.org/10.1109/TAP.2004.840528

PUBLISHER

(C) Institute of Electrical and Electronics Engineers (IEEE)

VERSION

VoR (Version of Record)

LICENCE

CC BY-NC-ND 4.0

\section{REPOSITORY RECORD}

Feresidis, Alexandros P., George Goussetis, Shenhong Wang, and J.C. Vardaxoglou. 2019. "Artificial Magnetic Conductor Surfaces and Their Application to Low-profile High-gain Planar Antennas". figshare. https://hdl.handle.net/2134/9424. 
This item was submitted to Loughborough's Institutional Repository (https://dspace.lboro.ac.uk/) by the author and is made available under the following Creative Commons Licence conditions.

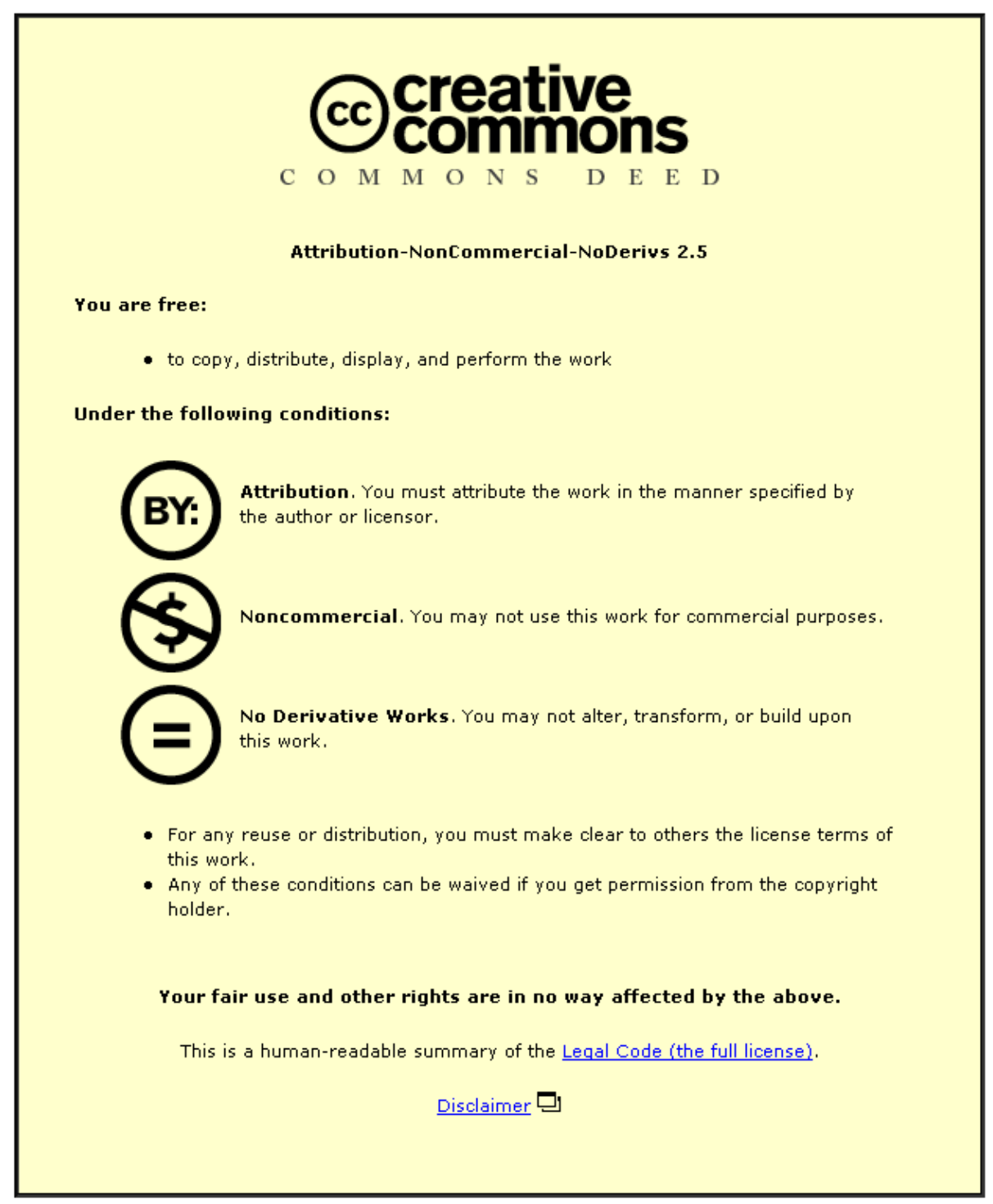

For the full text of this licence, please go to: http://creativecommons.org/licenses/by-nc-nd/2.5/ 


\title{
Artificial Magnetic Conductor Surfaces and Their Application to Low-Profile High-Gain Planar Antennas
}

\author{
Alexandros P. Feresidis, Member, IEEE, George Goussetis, Member, IEEE, Shenhong Wang, and \\ John (Yiannis) C. Vardaxoglou, Member, IEEE
}

\begin{abstract}
Planar periodic metallic arrays behave as artificial magnetic conductor (AMC) surfaces when placed on a grounded dielectric substrate and they introduce a zero degrees reflection phase shift to incident waves. In this paper the AMC operation of single-layer arrays without vias is studied using a resonant cavity model and a new application to high-gain printed antennas is presented. A ray analysis is employed in order to give physical insight into the performance of $\mathrm{AMCs}$ and derive design guidelines. The bandwidth and center frequency of AMC surfaces are investigated using full-wave analysis and the qualitative predictions of the ray model are validated. Planar AMC surfaces are used for the first time as the ground plane in a high-gain microstrip patch antenna with a partially reflective surface as superstrate. A significant reduction of the antenna profile is achieved. A ray theory approach is employed in order to describe the functioning of the antenna and to predict the existence of quarter wavelength resonant cavities.
\end{abstract}

Index Terms-Arrays, Artificial magnetic conductors, electromagnetic bandgap structures, high-gain antennas, low-profile antennas.

\section{INTRODUCTION}

$\mathbf{O}$ VER the last few years the electromagnetic band gap (EBG) properties of passive metallodielectric arrays have been studied [1]-[4]. The presence of band gaps similar to those obtained from dielectric photonic crystals [5] has been demonstrated. Metallodielectric EBG (MEBG) structures have been used for suppression of surface waves and performance enhancement of printed antennas and circuits. In addition, MEBG arrays have been utilized as partially reflective superstrate layers for the gain enhancement of simple radiating sources, such as microstrip patches and waveguide apertures [6]-[8]. Similar implementations using dielectric EBG structures have also been presented and novel explanations have been given [9]-[11].

Recently metallic arrays printed on a grounded dielectric substrate and connected to the ground through vias have been presented as artificial magnetic conductors (AMCs) [12]. Such surfaces fully reflect incident waves with a near zero degrees reflection phase. AMC surfaces have also been produced from sim-

Manuscript received January 15, 2004; revised July 23, 2004. This work was supported by the Engineering and Physical Sciences Research Council (EPSRC) of the U.K., under research grant GR/R42580/01.

The authors are with the Wireless Communications Research Group, Department of Electronic and Electrical Engineering, Loughborough University, Loughborough, LEll 3TU, U.K. (e-mail: a.feresidis@ ieee.org).

Digital Object Identifier 10.1109/TAP.2004.840528 ilar structures in the absence of vias, which eases the fabrication process [13], [14]. Assuming no losses and exactly $0^{\circ}$ reflection phase, the surface is referred to as a perfect magnetic conductor (PMC), which is complementary to a perfect electric conductor (PEC). In practice, the reflection phase of AMCs crosses zero at just one frequency (for one resonant mode). The useful bandwidth of an AMC is in general defined as $+90^{\circ}$ to $-90^{\circ}$ on either side of the central frequency, since these phase values would not cause destructive interference between direct and reflected waves.

Metallic arrays have also been placed over a ground plane in order to significantly enhance the directivity of simple radiating sources positioned between the array and the ground [6]-[8]. The structure is based on the formation of a resonant cavity between the ground plane and the array that acts as a partially reflective surface (PRS). While high gain planar antenna designs have been produced, the antenna profile, which is determined by the resonance condition of the cavity, has always been close to half wavelength.

In this paper, a novel resonant cavity approach to the analysis of AMCs is initially presented using ray theory. This provides design guidelines and a physical insight into the function of AMCs. Subsequently, a novel high-gain microstrip patch antenna with PRS superstrate is presented, where an AMC is utilized as ground plane to reduce the antenna profile. The antenna is also studied using a resonant cavity model with the source internal to the cavity. By virtue of the AMC ground plane the resonance condition changes and the thickness of the cavity is reduced to approximately half. The AMCs considered are comprised of a single layer array of patches (in the absence of vias) printed on a grounded dielectric substrate. Full-wave analysis and measurements of both the AMC alone and the antenna are used to validate the assertions of the cavity model.

The organization of the paper is as follows. In Section II a brief outline of the ray analysis is given and the existence of quarter wavelength resonant PEC-PMC cavities is predicted. The ray model for an AMC cavity is also described. In Section III, an explanation of AMC surfaces as resonant cavities is given. Using the ray model, the AMC response is related to the transmission characteristics of the array alone. A full-wave study on the AMC performance of square patch arrays is presented and the ray model predictions are validated. The AMC bandwidth and the effect of substrate thickness are studied. Section IV presents a new application of AMC ground planes to low profile high-gain antennas. The existence of a quarter wave- 


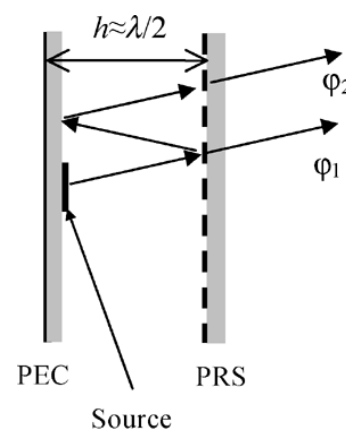

(a)

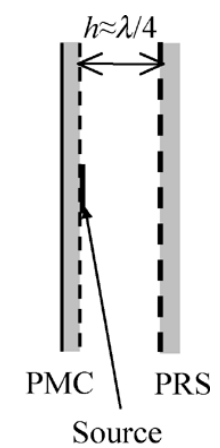

(b)
Fig. 1. Resonant cavity formed by (a) PEC and PRS, (b) PMC and PRS, with excitation inside the cavity.

length resonant cavity antenna predicted by the ray model is confirmed by full-wave analysis and measurements.

\section{ANALYSIS}

A simple geometrical optics model can be used to describe the function of resonant cavities. The ray optics analysis is widely used in the description of the Fabry-Perot interferometer, which consists of two highly reflective surfaces that form a resonant cavity [15]. This analysis can be carried over to the design of high-gain microwave antennas. In the past, highly-directive planar antennas consisting of a ground plane, a simple radiating source and a partially reflective surface (PRS) as superstrate have been studied using this method [6]-[8]. In these studies, surfaces of infinite extent have been assumed (i.e., no edge effects) and higher order mode coupling has been ignored. Although an approximate analysis, it gave a valuable insight to the function of highly-directive antennas as resonant cavities and produced guidelines for successful designs. The analysis is briefly outlined in this section. It is then used to theoretically predict the existence of a low-profile highly-directive antenna as a quarter wavelength (instead of half wavelength) resonant cavity by using a PMC (instead of PEC) ground plane. In addition, the same ray model is used in order to obtain an explanation of the function of an AMC structure as a resonant cavity. For the first case the excitation source is considered inside the resonant cavity whereas for the second case the source is outside the cavity.

The structure shown in Fig. 1(a) is considered. A cavity is formed by a PEC ground plane and a PRS placed at a distance $h$. The PRS is assumed to be a homogeneous surface in the analysis. The antenna function can be described by following the paths of the waves undergoing multiple reflections inside the cavity. Phase shifts are introduced by the path length, the PEC $(\pi)$ and the phase of the reflection coefficient of the PRS. The transmitted power can be derived by considering the interference of waves partially transmitted through the PRS. Hence, the directivity of the cavity at boresight is given by [6]-[8]

$$
D=\frac{P_{T}}{P_{0}}=\frac{\left[1-R^{2}\right]}{1+R^{2}-2 R \cos [\Delta \phi]}
$$

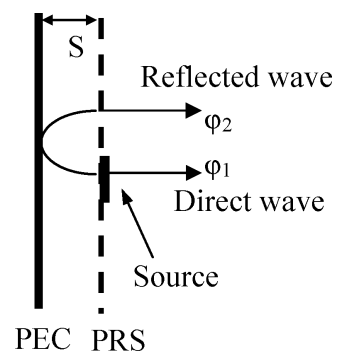

Fig. 2. Resonant cavity formed by PEC and PRS with excitation outside the cavity.

where $R e^{j \phi_{R}}$ is the complex reflection coefficient of the PRS, $\Delta \phi=\phi_{2}-\phi_{1}, \lambda$ is the free-space wavelength, $P_{T}$ is the transmitted power and $P_{0}$ the power of the excitation source. The resonance condition can be easily derived by imposing the phase difference of the transmitted waves to be zero, $\Delta \varphi=2 N \pi$, and is written

$$
\begin{aligned}
\Delta \phi & =\phi_{R}-\pi-\frac{2 \pi}{\lambda} 2 h=2 N \pi, \quad N=0,1,2 \ldots \\
\text { or } h & =\left(\frac{\phi_{R}}{\pi}-1\right) \frac{\lambda}{4}+N \frac{\lambda}{2} .
\end{aligned}
$$

At resonance maximum boresight directivity is obtained and it can be derived by substituting (2) into (1)

$$
D_{\max }=\frac{1+R}{1-R} \text {. }
$$

This equation expresses the maximum directivity as a function of the magnitude of the reflection coefficient of the PRS. From the above analysis it can be derived that for a highly reflective surface used as the PRS, with reflection phase close to $\pi$, the thickness of the resonant cavity is close to $\lambda / 2$. For this cavity, the directivity as obtained from (3) will be very high, since $R$ will be close to 1 .

If the PEC in the cavity is now replaced by another fictitious ground plane which introduces a phase shift $\psi_{R}$ to incident waves, the resonance condition becomes

$$
\phi_{R}+\psi_{R}-\frac{2 \pi}{\lambda} 2 h=2 N \pi, \quad N=0,1,2 \ldots
$$

Hence, if the ground plane is a PMC [Fig. 1(b)], i.e., if $\psi_{R}=0$, the resonance condition becomes

$$
h=\frac{\phi_{R}}{\pi} \frac{\lambda}{4}+N \frac{\lambda}{2}, \quad N=0,1,2 \ldots
$$

Therefore, a PMC surface with reflection phase zero and a highly reflective PRS (with $\phi_{R}=\pi$ ) would result in a resonant cavity of thickness approximately $\lambda / 4$. The above analysis thus predicts the profile reduction of resonant cavity antennas to half, by means of replacing the PEC with a PMC ground plane.

Consider now the case where a radiating source is placed outside the cavity adjacent to the PRS array (Fig. 2). Following the paths of the direct and the reflected waves and taking into account the various phase shifts introduced to them, the resonance condition of the cavity can be easily derived. The PEC introduces a phase shift of $\pi$. The PRS introduces a phase shift equal to the phase of its transmission coefficient, $\varphi_{\mathrm{T}}$. If $\varphi_{2}-\varphi_{1}$ 


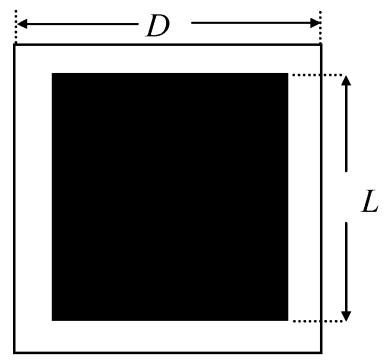

(a)

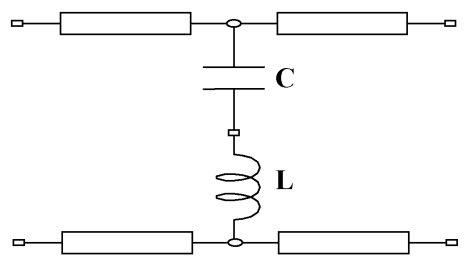

(b)
Fig. 3. (a) Unit cell of square patch array and (b) typical equivalent circuit for capacitive screen consisting of rectangular patches.

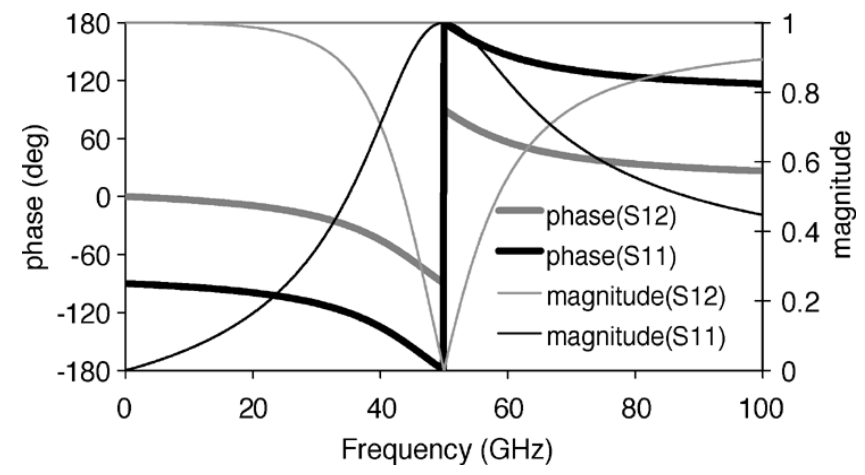

Fig. 4. Transmission/reflection phase and magnitude for PRS equivalent circuit (Fig. 3).

is the phase difference between direct and reflected waves, the resonance condition is written as follows:

$$
\phi_{2}-\phi_{1}=2 \phi_{T}-\frac{2 \pi}{\lambda} 2 S-\pi=2 N \pi, \quad N=0,1,2 \ldots
$$

This resonant cavity behaves as a PMC (at normal incidence) since it reflects normal incident waves with zero phase shift. Consequently, placing a simple point source in close proximity to the PRS would result in constructive interference between direct and reflected waves at the cavity resonance.

\section{PlanAR AMC SURFACES}

In this section the resonant cavity model is initially employed to provide with guidelines on the design of AMCs. Full-wave Floquet modal analysis [3] of periodic metallodielectric PRS surfaces and AMC structures without vias is carried out in order to validate the conclusions obtained from the approximate cavity model. The bandwidth of AMC surfaces is studied and the effect of the substrate thickness is presented.

\section{A. AMC as Resonant Cavity}

A homogenised model for a capacitive PRS screen [Fig. 3(a)] is employed here to facilitate the analysis. The equivalent circuit representation of the PRS is shown in Fig. 3(b), where the conducting element is represented by the inductor $\mathbf{L}$ and the inter-element capacitance by the capacitor $\mathbf{C}$. The complex reflection and transmission coefficients obtained from the equivalent circuit are shown in Fig. 4. Similar responses are obtained

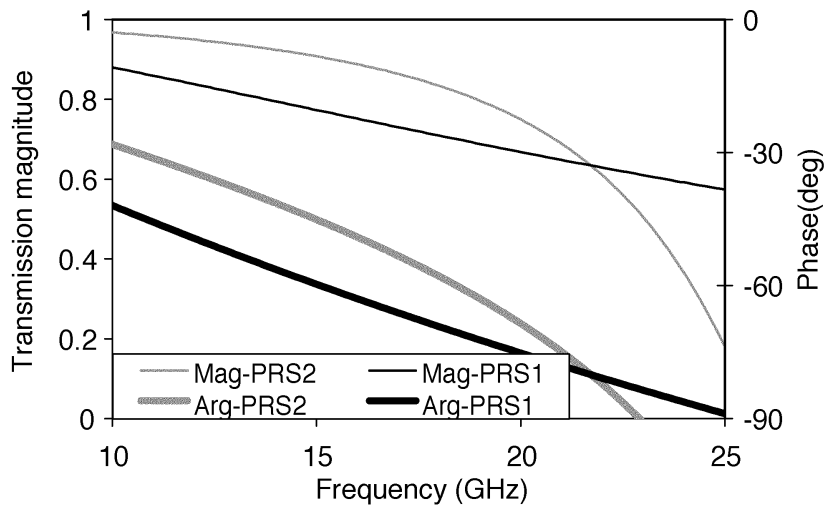

(a)

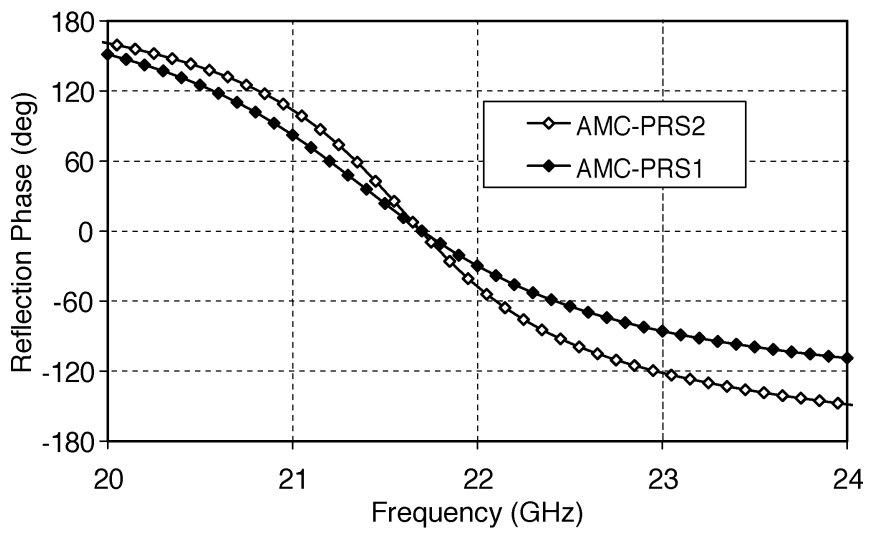

(b)

Fig. 5. (a) Simulated transmission magnitude and phase of PRS1 and PRS2 (b) AMC reflection responses for same cavity with PRS1 and PRS2, respecively.

from full-wave Floquet modal analysis of infinite periodic arrays of conducting elements. While this model does not account for all the geometrical parameters of the PRS, it is a good representation for a wide range of geometries.

According to the ray model presented in Section II (Fig. 2), a cavity formed by a PEC and a PRS and having external excitation performs as AMC when the resonance condition (5) is met. Hence, considering (5) as the condition for AMC operation (normal incidence), a relationship between the transmission phase of the PRS, the substrate thickness and the center (or PMC) operating frequency is obtained.

The relation between the PRS characteristics and the functioning of the AMC cavity is demonstrated by means of an example which shows that two different PRS's having same reflection and transmission characteristics at frequency $f_{o}$ are interchangeable in an AMC cavity that operates at $f_{o}$. Fig. 5(a) shows the reflection coefficient (magnitude and phase) of two capacitive screens consisting of square patch arrays [Fig. 3(a)]. The geometries of the two arrays are: $L=4.15 \mathrm{~mm}, D=4.50 \mathrm{~mm}$ for the first screen named PRS1 and $L=6.00 \mathrm{~mm}, D=10.00 \mathrm{~mm}$ for the second screen named PRS2. PRS1 resonates at $60.5 \mathrm{GHz}$ and PRS2 at $26.0 \mathrm{GHz}$. The reflectivity and transmission phase at $21.7 \mathrm{GHz}$ is identical for the two screens. Fig. 5(b) shows the full wave simulation results for two AMC cavities of the same thickness $S$ employing PRS1 and PRS2, respecively. The thickness $S$ has been determined from (5) so that the AMC cavities operate at $21.7 \mathrm{GHz}$. In order to have good agreement between 
the ray model and the full-wave results, we are working at the second $(N=1)$ rather than the first $(N=0)$ resonant mode of the cavity [see (5)]. As predicted by the ray model, the full wave AMC responses are centred at the same frequency $21.7 \mathrm{GHz}$, where the transmission phase values are common. For different cavity thickness, each PRS results in different AMC center frequency.

It is worth noting that according to (5), the second resonant mode of the AMC cavity lies at a frequency approximately three times that of the first resonant mode. Thus, the resonant cavity model provides a new explanation for the large separation between the first and second AMC frequencies of grounded square patch arrays that has also been studied in [14]. Moreover, the low reflection magnitude observed at the second AMC frequency can be explained by the fact that for increasing $N$ the sidelobes of the resonant cavity radiation pattern increase, as described in [6], [7].

\section{B. Bandwidth Considerations}

According to (5), for a wideband AMC, an optimum PRS would require its transmission coefficient phase to linearly increase with frequency with a gradient of $2 \pi S / \mathrm{c}$ [8]. This would result in a wideband cavity that would satisfy the resonance condition (5) for all frequencies. While increasing transmission phase with frequency is not feasible for a capacitive screen, this conclusion suggests that among two screens with equal reflectivity, greater AMC bandwidth will be observed for the one with slower varying transmission phase. This is demonstrated using full wave results in the example mentioned above (Fig. 5). PRS1 has slower transmission phase variation with frequency compared to PRS2 [Fig. 5(a)]. The AMC bandwidth for PRS1 is $30 \%$ wider than that of PRS2, for the same substrate thickness [Fig. 5(b)].

\section{Effect of Substrate Thickness}

The resonant cavity AMC model predicts that for a fixed capacitive screen with an approximately frequency independent phase, the AMC frequency decreases as the dielectric thickness increases. Furthermore, from Fig. 4 it is evident that away from the array resonance, the PRS phase variation with frequency is slower, which in turn corresponds to broader AMC bandwidth. Therefore, in general, with increasing $S$, the AMC frequency decreases and the bandwidth is improved. This is also consistent with the analysis of reflectarray antennas [16].

The effect of substrate thickness is demonstrated here in Fig. 6, using full-wave analysis. A parametric study of the AMC response for a fixed square patch PRS is presented. The dielectric constant of the substrate is 2.2. The trends predicted from the approximate model are verified. The frequency of the AMC operation is reduced and the bandwidth is improved with thicker dielectric substrates.

\section{Profile Reduction of High-Gain Antenna}

The application of AMC surfaces to low-profile high-gain antennas is presented in this section. A broadband AMC surface is designed based on the methodology of the previous section and is used as the ground plane of a microstrip patch antenna with

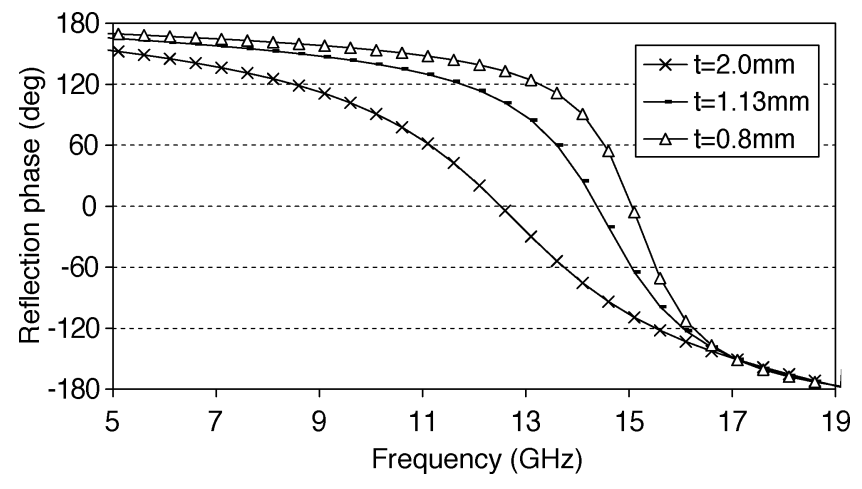

Fig. 6. Parametric study of AMC performance with thickness t of dielectric slab.

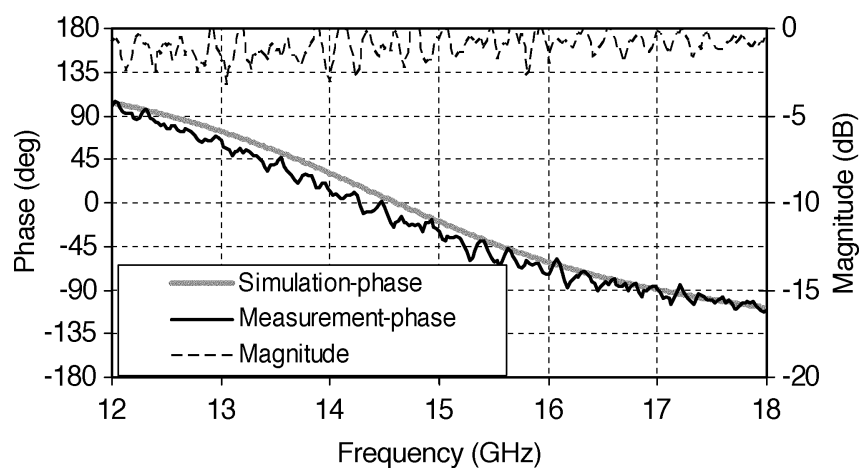

Fig. 7. Reflection magnitude and phase of AMC ground plane (normal incidence).

a PRS superstrate. According to the analysis in Section II the AMC ground plane in conjunction with a highly reflective PRS superstrate are expected to produce a low-profile (about quarter wavelength) high-gain planar antenna. Full wave simulations of the antenna have been carried out prior to the fabrication stage and are in good agreement with measured results.

\section{A. Design of AMC Substrate and PRS Superstrate}

A broadband AMC surface has been designed with a center frequency (zero degrees phase) at about $14 \mathrm{GHz}$. The simulated reflection phase response as obtained from Floquet modal analysis is shown in Fig. 7. Closely packed square patches were used with unit cell dimensions $L=4.1 \mathrm{~mm}, D=4.5 \mathrm{~mm}$ [Fig. 3(a)]. The patches were printed on a grounded dielectric slab of thickness $\mathrm{S}_{\mathrm{sub}}=1.13 \mathrm{~mm}$ and $\varepsilon_{\mathrm{r}}=2.2$. The measured complex reflection coefficient of the fabricated surface has been obtained with reference a solid metal plate at the position of the array and is shown in Fig. 7. The measured reflection magnitude is close to 1 as expected from an AMC surface. The simulation is in good agreement with the measured phase response. A broadband AMC operation is obtained with a $+90^{\circ}$ to $-90^{\circ}$ bandwidth of more than $30 \%$ (about $4.5 \mathrm{GHz}$ ).

A highly reflective PRS has been produced using a square patch array printed on a similar dielectric substrate as the AMC (without the ground plane). The unit cell dimensions are $L=$ $10 \mathrm{~mm}, D=11 \mathrm{~mm}$. The complex reflection coefficient of the PRS obtained from modal analysis is shown in Fig. 8. High reflectivity values are obtained for a wide range of frequencies. Moreover, the slow variation of the reflection phase con- 


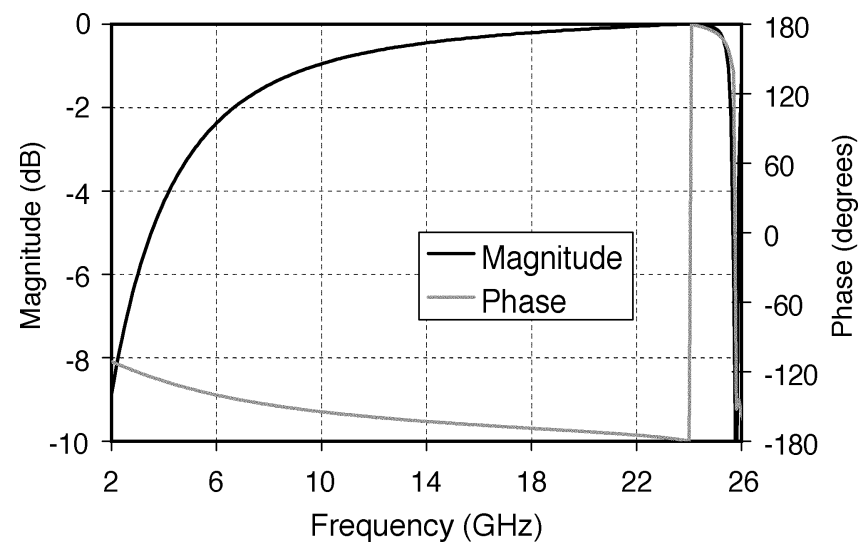

Fig. 8. Simulated complex reflection coefficient of PRS superstrate (normal incidence).

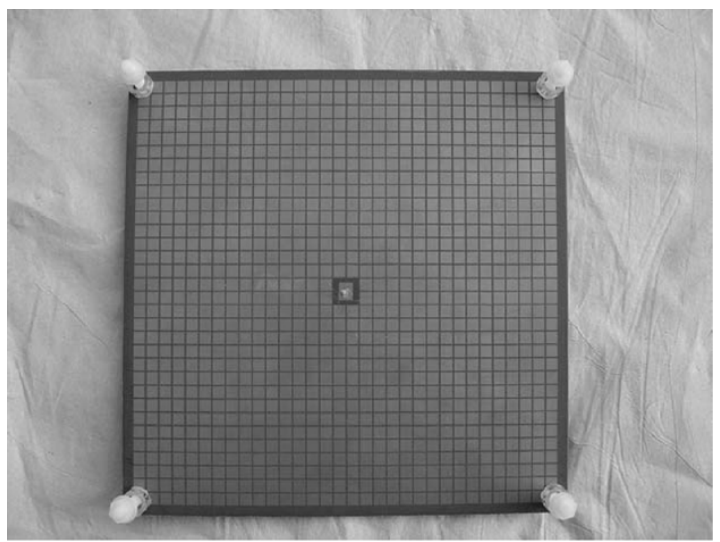

(a)

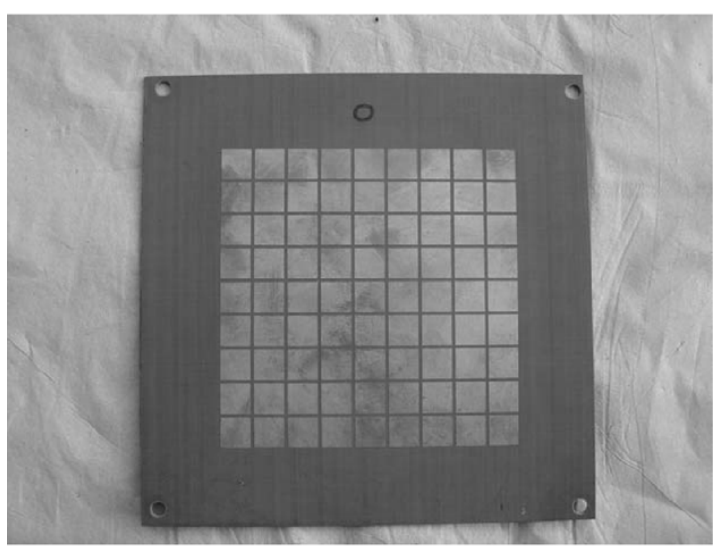

(b)

Fig. 9. Photographs of (a) patch antenna with AMC ground plane and (b) PRS superstrate.

tributes toward a good bandwidth performance for the antenna according to the ray model in Section II and in [8]. The reflection coefficient at $14 \mathrm{GHz}$ is about $-0.43 \mathrm{~dB}$. Following (3) of the ray model a maximum antenna directivity of about $20.5 \mathrm{dBi}$ is expected.

\section{B. Antenna Performance}

A microstrip patch antenna $(6 \mathrm{~mm} \times 4.5 \mathrm{~mm})$ has been designed as the primary feeder of the resonant cavity and fabricated using the AMC ground plane $\left(15 \times 15 \mathrm{~cm}^{2}\right)$. The patches

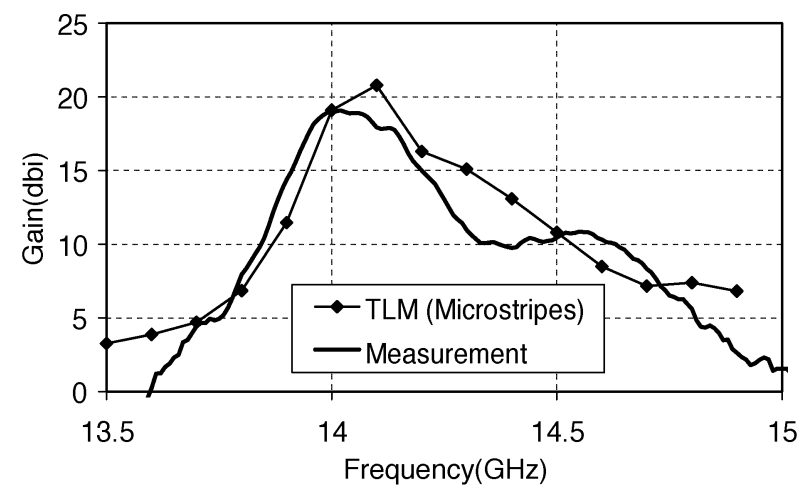

(a)

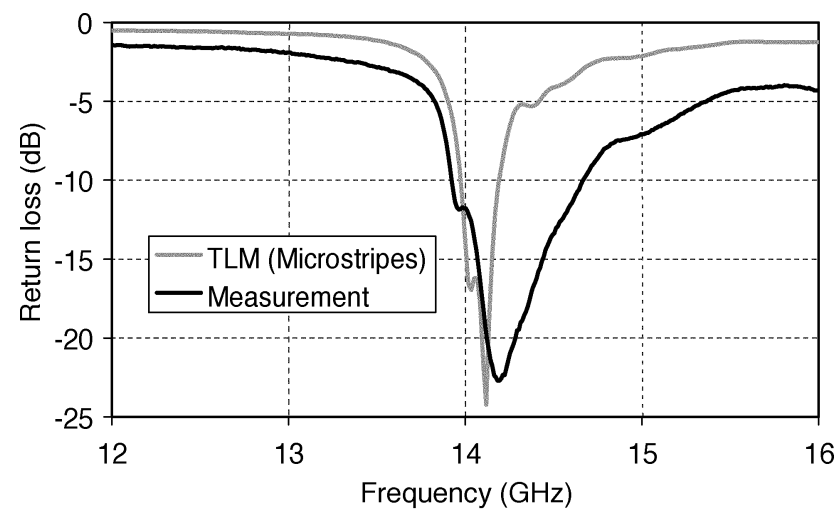

(b)

Fig. 10. (a) Gain and (b) return loss of low-profile planar antenna.

of the AMC are surrounding the patch antenna which is printed on the same dielectric substrate. It is fed by a via which is 1 $\mathrm{mm}$ offset from the center of the patch, in order to achieve good matching. The AMC does not extend under the patch antenna since it was found that matching was very difficult for this configuration. The design of the antenna was carried out using a 3-D time domain Transmission Line Modeling (TLM) tool (Microstripes). Fig. 9(a) shows a photograph of the fabricated patch antenna with the AMC and Fig. 9(b) shows the PRS superstrate.

The high-gain planar antenna is formed by placing the PRS at the resonant distance over the microstrip patch with the AMC ground plane. The resulting structure is similar to the schematic diagram of Fig. 1(b) in Section II. A full-wave simulation of the antenna was carried out in Microstripes. The high-gain antenna was fabricated and measured. The measured resonant distance between the PRS superstrate and the AMC array was found to be $5.9 \mathrm{~mm}$. This corresponds to an approximately quarter wavelength resonant cavity at $14 \mathrm{GHz}$ and agrees very well with the value obtained from (4), (5.85 mm). The corresponding resonant distance for the same antenna configuration but with a PEC (instead of the AMC) ground plane was obtained from 3-D simulations as well as the ray model to be $11.3 \mathrm{~mm}$. Therefore, a reduction of the antenna profile by approximately half has been achieved by virtue of employing the AMC ground plane.

The gain of the low-profile antenna is shown in Fig. 10(a). A maximum gain of $19 \mathrm{dBi}$ has been obtained. Simulation results are in good agreement with measurements. The maximum directivity predicted by the ray model $(20.5 \mathrm{dBi})$ is also in good agreement. The return loss of the antenna is depicted in 


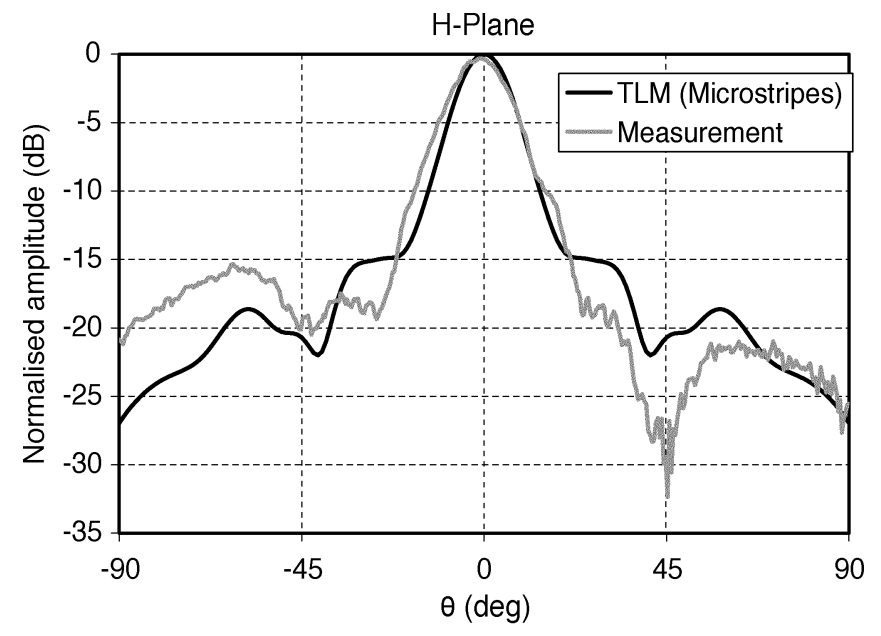

(a)

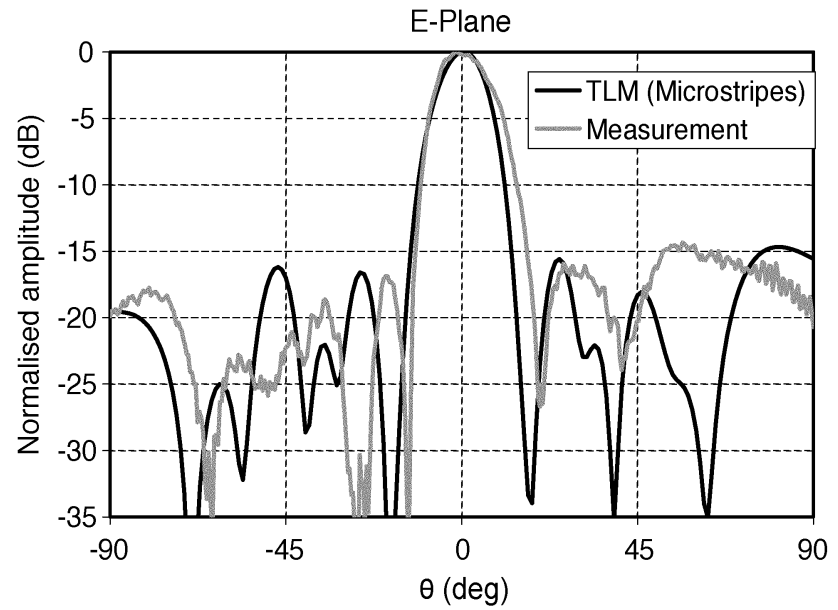

(b)

Fig. 11. Radiation patterns of low-profile antenna (a) H plane and (b) E plane.

Fig. 10(b). The measured antenna bandwidth as defined from the $-3 \mathrm{~dB}$ gain level and $10 \mathrm{~dB}$ return loss is about $2 \%$. The maximum gain obtained from the corresponding higher profile antenna with a PEC ground plane is about the same.

Both the $\mathrm{H}$ - and E-plane radiation patterns are shown in Fig. 11. In both planes highly directive pattern is obtained and the sidelobe level is below $-15 \mathrm{~dB}$. The small discrepancies between simulation and measurements are attributed to the manufacturing tolerances of the low-profile antenna with regards to accurate positioning and the flatness of the surfaces. The cross polarization level was below $-25 \mathrm{~dB}$ in both planes.

\section{CONCLUSION}

A resonant cavity model has been proposed in order to describe the functioning of AMC surfaces. Using a simple analytical equation derived from ray theory, the AMC response has been related to the transmission characteristics of the PRS array alone. While this model does not account for accurate design of AMCs, which at the final stage has to be made using full-wave techniques, it provides with valuable physical insight and design guidelines. Full wave results validated the trends predicted from the ray model, with regard to the bandwidth and the center frequency.

A new application of AMC ground planes to low-profile highgain planar antennas with PRS superstrate has been presented. A ray model has provided valuable insight into the function of the antenna as a resonant cavity and moreover predicted the existence of quarter wavelength PEC-PMC resonant cavities. The antenna profile has been reduced to approximately half, maintaining the gain performance. Measured and simulated results have been presented and are in good agreement.

\section{ACKNOWLEDGMENT}

The authors acknowledge the technical support of Mr. N. Carpenter.

\section{REFERENCES}

[1] P. De Maagt, R. Gonzalo, J. C. Vardaxoglou, and J. M. Baracco, "Photonic bandgap antennas and components for microwave and (sub)millimeter wave applications," Special Issue on Metamaterials IEEE Trans. Antennas Propag., vol. 51, no. 10, pp. 2667-2677, Oct. 2003.

[2] Y. R. Lee, A. Charaya, D. S. Lockyer, and J. C. Vardaxoglou, "Dipole and tripole metallodielectric photonic bandgap (MPBG) structures for microwave filter and antenna applications," Proc. Inst. Elect. Eng. Optoelectron., vol. 127, no. 6, pp. 395-400, Dec. 2000.

[3] A. P. Feresidis, G. Apostolopoulos, N. Serfas, and J. C. Vardaxoglou, "Closely Coupled Metallodielectric Electromagnetic Band Gap (CCMEBG) structures formed by double layer dipole and tripole arrays," IEEE Trans. Antennas Propag., vol. 52, no. 5, pp. 1149-1158, May 2004.

[4] R. Abhari and G. V. Eleftheriades, "Metallo-dielectric electromagnetic bandgap structures for suppression and isolation of the parallel-plate noise in high-speed circuits," IEEE Trans. Microw. Theory Tech., vol. 51, no. 6, pp. 1629-1639, Jun. 2003.

[5] R. Gonzalo, P. de Maagt, and M. Sorolla, "Enhanced patch antenna performance by suppressing surface waves using photonic bandgap substrates," IEEE Trans. Microw. Theory Tech., vol. 47, no. 11, pp. 2131-2138, Nov. 1999.

[6] G. V. Trentini, "Partially reflecting sheet arrays," IRE Trans. Antennas Propag., vol. AP-4, pp. 666-671, 1956.

[7] J. R. James, S. J. A. Kinany, P. D. Peel, and G. Andrasic, "Leaky-wave multiple dichroic beamformers," Electron. Lett., vol. 25, 1989.

[8] A. P. Feresidis and J. C. Vardaxoglou, "High gain planar antenna using optimized partially reflective surfaces," Proc. Inst. Elect. Eng. Microw. Antennas Propag., vol. 148, no. 6, pp. 345-350, Dec. 2001.

[9] D. R. Jackson and N. G. Alexopoulos, "Gain enhancement methods for printed-circuit antennas," IEEE Trans. Antennas Propag., vol. AP-33, no. 9, pp. 976-987, Sep. 1985.

[10] D. R. Jackson and A. A. Oliner, "A leaky-wave analysis of the high-gain printed antenna configuration," IEEE Trans. Antennas Propag., vol. 36, no. AP-7, pp. 905-910, Jul. 1988.

[11] C. Cheype, C. Serier, M. Thevenot, T. Monediere, A. Reineix, and B. Jecko, "An electromagnetic band gap resonator antenna," IEEE Trans. Antennas Propag., vol. 50, no. 9, pp. 1285-1290, Sep. 2002.

[12] D. Sievenpiper, L. Zhang, R. F. J. Broas, N. G. Alexopolous, and E. Yablonovitch, "High-impedance electromagnetic surfaces with a forbidden frequency band," IEEE Trans. Microw. Theory Tech., vol. 47, no. 11, pp. 2059-2074, Nov. 1999.

[13] Y. E. Erdemli, K. Sertel, R. A. Gilbert, D. E. Wright, and J. L. Volakis, "Frequency-selective surfaces to enhance performance of broad-band reconfigurable arrays," IEEE Trans. Antennas Propag., vol. 50, no. 12, pp. 1716-1724, Dec. 2002.

[14] Y. Zhang, J. von Hagen, M. Younis, C. Fischer, and W. Wiesbeck, "Planar artificial magnetic conductors and patch antennas," Special Issue on Metamaterials, IEEE Trans. Antennas Propag., vol. 51, no. 10, pp. 2704-2712, Oct. 2003.

[15] J. T. Verdeyen, Laser Electronics. Englewood Cliffs, NJ: Prentice-Hall, 1989, pp. 123-131.

[16] D. M. Pozar, S. D. Targonski, and H. D. Syrigos, "Design of millimeter wave microstrip reflectarray," IEEE Trans. Antennas Propag., vol. 45, no. 2, pp. 287-295, Feb. 1997. 


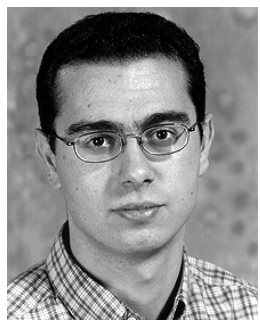

Alexandros P. Feresidis (M'98) was born in Thessaloniki, Greece, in 1975. He received the B.Sc. (Ptychio) degree in physics from Aristotle University of Thessaloniki (AUTH), in 1997, the M.Sc. (Eng.) degree in radio communications and high-frequency engineering from the University of Leeds, Leeds, U.K., in 1998, and the Ph.D. degree in electronic and electrical engineering from Loughborough University, Leicestershire, U.K., in 2002.

During the first half of 2002, he was a Research Associate with the Wireless Communications Research Group, Department of Electronic and Electrical Engineering, Loughborough University. He is currently a Lecturer in the same department. His research interests include analysis and design of EBG materials and frequency selective surfaces (FSS), periodic structures, high-gain array antennas, base station antennas, evolutionary computation techniques in electromagnetics, and microwave circuits.

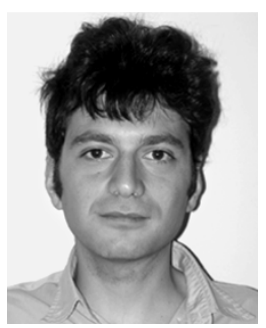

George Goussetis (M'02) was born in Athens, Greece, in 1976. He received the Electrical and Computer Engineering degree from the National Technical University of Athens, in 1998, the B.Sc. degree in physics from University College London (UCL), London, U.K., in 2002, and the Ph.D. degree in the area of waveguide filters from the University of Westminster, London, in 2002.

In 1999, he joined the Wireless Communications Research Group, University of Westminster, as a Research Assistant. In 2002, he joined the wireless communications research group, Loughborough University, U.K., where he is currently employed as a Senior Research Fellow. His research interests include the modeling and design of microwave filters and passive components, EBG materials and periodic structures.

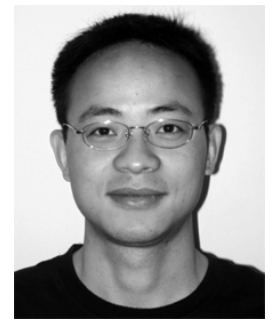

Shenhong Wang was born in Jiangsu, China. He received the B.S. degree in communications engineering and the M.S. degree in Communications System from Nanjing University of Posts and Telecommunications, Nanjing, China, in 1996 and 1999 , respectively. He is currently working toward the Ph.D. degree in the Department of Electronic and Electrical Engineering, Loughborough University, Leicestershire, U.K.

From May 1999 to February 2000, he was a Research Assistant at Great Dragon IT Ltd., where he worked on PSTN-IP Intelligent Network Platform (partially funded by the China 863 High-Tech Program). From March 2000 to November 2002, he was a Software Engineer at Motorola, China, working on Call Processing of GSM/CDMA2000. Since December 2002, he has been with the Wireless Communications Group, Department of Electronic and Electrical Engineering, Loughborough University. His current research interests mainly focus on low-profile high-gain planar antennas and metamaterials (FSS, AMC, and EBG).

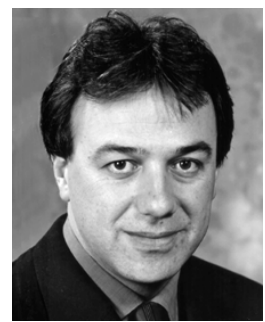

John (Yiannis) C. Vardaxoglou (M'87) received the B.Sc. degree in mathematics (mathematical physics) and the Ph.D. degree from the University of Kent at Canterbury, U.K., in 1981 and 1985, respectively.

In January 1988, he was appointed Lecturer in Communications with the Department of Electronic and Electrical Engineering, Loughborough University of Technology, U.K. He was promoted to the position of Senior Lecturer in January 1992. In 1998, he was appointed Professor of Wireless Communications. He holds the Chair of Wireless Communications at Loughborough University and is the Founder of the Centre for Mobile Communications Research (CMCR). He established the Antennas and Microwaves Research Group at Loughborough University and he heads the Centre for Mobile Communications Research (CMCR). He has been active in the area of electromagnetic modeling and applications of frequency selective surfaces (FSS). His current research interests include wireless communication networks, array antennas, FSS, radomes, leaky-wave resonant antennas, optical control of microwaves and devices, periodic surfaces and EBG/PBG materials, and mobile telephone antennas. His contribution to the CMCR lies in the analysis and design of small-loaded antennas for mobile telephony. He has served as a Consultant to various industries in the U.K. and abroad. He holds three patents and is the Technical Director of Antrum Ltd. He has published over 120 refereed journals and conference proceeding papers and has written a research monograph on frequency selective surfaces.

Dr. Vardaxoglou is currently a Member of the executive committee of the Institution of Electrical Engineers (IEE), London, U.K., Professional Network in Antennas and Propagation. He chaired the 1st IEE Antenna Measurements and SAR (AMS'02) conference and has been on the organizing committee of the 2001 and 2003 IEE International Conferences on Antennas and Propagation. 